\title{
EFFECT OF PRE-FRACTURE MOBILITY ON THE EARLY POST-OPERATIVE FUNCTIONAL OUTCOME IN ELDERLY PATIENTS WITH A HIP FRACTURE
}

\begin{abstract}
Background: Hip fractures are among the most common causes of disability and hospitalisation in the elderly. There are no studies in South Africa that determine the effect of pre-fracture functional mobility on early post-operative functional outcome in elderly patients with a hip fracture. Aim: The aim of this study was to determine the effect of pre-fracture functional mobility on early post-operative functional outcome in elderly patients with a hip fracture.
\end{abstract}

Methodology: A prospective pre-test post-test observational study design was done. Assessments were conducted pre-operatively, at discharge and six weeks post discharge at two public hospitals in Johannesburg, South Africa. The pre-fracture functional mobility of the participants was determined using the New Mobility Score (NMS) pre-operatively. At discharge and at six weeks post discharge the participants post-operative functional level was assessed using the Elderly Mobility Scale (EMS) and the Lower Extremity Functional Scale (LEFS).

Results: More than two thirds of participants were independently mobile prior to the fracture. Pre-fracture functional mobility is a strong determinant of early post operative functional outcome in elderly patients with a hip fracture $(\beta=1.39, p=0.0001)$.

Conclusion: Independent pre-fracture mobility predicts better early post-operative functional outcomes in the elderly.

KEY WORDS: HIP FRACTURE; ELDERLY PATIENTS, PRE-FRACTURE FUNCTIONAL MOBILITY, EARLY POST-OPERATIVE FUNCTIONAL OUTCOME.

\section{INTRODUCTION}

Hip fractures are among the most common causes of disability and hospitalisation in the elderly and result in significant morbidity and mortality (Williams et al, 2004). The incidence of falls rises steadily after the age of 65 due to increasing age and frailty and occurs in 30 to $60 \%$ of elderly persons annually (WHO, 2007). The ability of a patient to walk prior to the fracture is a good indicator of the general medical and mental health of the patient (Holt et al, 1994).

\section{Correspondence Author:}

Saadiya Adam

Helen Joseph Hospital

Auckland Park

Johannesburg, South Africa.

Email: adam.saadiya@gmail.com
Pre-fracture functional level is known to be a predictive factor of rehabilitation outcome (Hershkovitz et al, 2007). The functional level before the fracture appears to be the most consistent predictor of short-term rehabilitation outcome and 30 day mortality in hip fractures in the elderly (Kristensen et al, 2010; Foss et al, 2006). Kristensen et al, (2010) concluded that a patient with a low pre-fracture functional level is 18 times more likely not to regain independence in basic mobility during hospitalisation or will regain independence in mobility on average three days later. Determining the influencing factors on early postoperative functional outcome in elderly patients with a hip fracture in a selected public health setting in South Africa will identify those patients who are especially at risk of not regaining independence in basic mobility. Exploration of this topic may lead to future studies on improving functional outcome for these high risk patients. Currently, there are no new studies available in South Africa that determine the factors that influence the early post-operative functional outcome in elderly patients with a hip fracture. The aim of this study was to establish the factors that influence the early postoperative functional outcome in elderly patients with a hip fracture.

\section{METHOD}

Study design and description of participants

A prospective pre-test post-test observational study was conducted. Elderly patientsover the age of 60 years with a first time unilateral hip fracture were consecutively sampled from the orthopaedic wards of two public 
health care hospitals in Johannesburg, South Africa. Patients with bilateral hip fractures, polytrauma and those with co-morbidities affecting mobility (CVA, Parkinsons disease or a spinal cord injury) were excluded. Patients re-admitted with complications of a previous surgery or those managed conservatively were also excluded.

The sample size for this study was determined using a power calculation on STATA. The power was set at $90 \%$ and alpha at 5\%. Standard deviations and the minimally clinical important difference for the Elderly Mobility Scale (EMS) and Lower Extremity Functional Scale (LEFS) were used. A loss to follow up ratio was taken into consideration and was set at $20 \%$, thus resulting in a final number of 90 subjects. Ethical clearance was granted by the Human Research Ethics Committee of the University of the Witwatersrand (M110403).

\section{PILOT STUDY}

A pilot study was carried out to determine the intra-rater reliability of the first author, to familiarise herself with the tools that were used in the main study and to establish the time taken to implement these tools. The patients were divided into three groups consisting of nine patients in each group. Each group was assessed pre-operatively, at discharge and six weeks post discharge. A total of 27 subjects participated in the pilot study.

The main study began after the pilot study was carried out. Ninety participants were evaluated. Pre-operatively, participants pre-fracture mobility was assessed using the New Mobility Score (NMS). Post-operative functional out come assessed using the EMS and LEFS was determined at discharge $(\mathrm{n}=82)$ and six weeks post discharge $(n=72)$. The percentage of participants who dropped out during the study was eight percent. At discharge, one participant withdrew consent due to reasons not explained to the first author. Six other participants did not arrive for the six week post discharge assessment. These participants could not be reached telephonically or physically.

Figure 1 depicts the follow up of participants during the study.

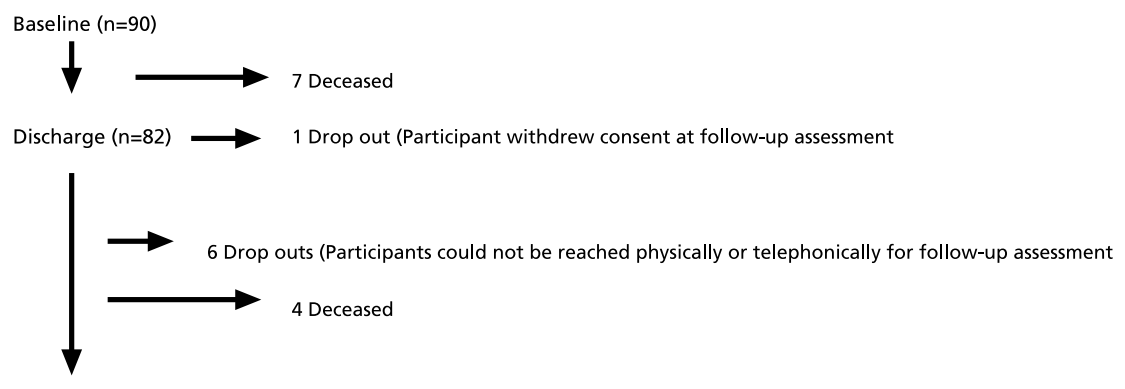

Six weeks post discharge $(n=72)$

Breakdown of 90 consecutive participants with a hip fracture, 72 of whom were included in the final analysis

Figure 1: Follow-up of participants during the study

Table 1: New Mobility Score(NMS) (Palmer and Parker, 1993)

\begin{tabular}{|l|l|l|l|l|}
\hline Mobility & $\begin{array}{l}\text { No } \\
\text { difficulty }\end{array}$ & $\begin{array}{l}\text { With an } \\
\text { aid }\end{array}$ & $\begin{array}{l}\text { With help } \\
\text { from another } \\
\text { person }\end{array}$ & Not at all \\
\hline $\begin{array}{l}\text { Able to get about the } \\
\text { house }\end{array}$ & 3 & 2 & 1 & 0 \\
\hline $\begin{array}{l}\text { Able to get out of the } \\
\text { house }\end{array}$ & 3 & 2 & 1 & 0 \\
\hline Able to go shopping & 3 & 2 & 1 & 0 \\
\hline
\end{tabular}

\section{OUTCOME MEASURES}

The New Mobility Score (NMS) (Table 1) was used pre-operatively to assess pre-fracture functional level. It is a composite score of the ability of a patient to perform indoor walking, outdoor walking and shopping prior to fracturing the hip. A score between zero and three is provided for each function (item) $($ zero $=$ not at all, one $=$ with help from another person, two $=$ with an aid, three $=$ no difficulty, no aid). The total score ranges from zero to nine. A score of zero indicates that the patient has no walking ability at all and a score of nine indicates full independence (Kristensen et al, 2010). The NMS has been shown to be both reliable and valid by Kristensen et al, (2008) and Parker and Palmer, (1993) respectively. In a study by Kristensen et al, (2008) a high inter-tester reliability score (0.98) was recorded, and this was evaluated using the intraclass correlation coefficient. A cut off score of five on the NMS is the best valid predictor of functional outcome at one year (Parker and Palmer, 1993). The NMS was administered using an interview.

The Elderly Mobility Scale (EMS) is a physical assessment of function and has a score out of 20. The EMS is an ordinal scale devised to provide a physiotherapy-orientated measure for frail elderly people which is complimentary to other scales (Smith, 1994). The scale assesses seven dimensions of functional performance. These include; locomotion, balance and key position changes, all of which are skills required for the performance of activities of daily living. The maximum score is 20 , with higher scores indicating better performance. Latent class analysis of the EMS showed strong inter-rater $(\rho=1.00)$ and intrarater reliability $(\rho=0.72)$. Concurrent validity was evaluated by a comparison to the Modified Rivermead Mobility Index. A high correlation between the two scales was revealed (Spearman's $\rho=0.89$ ), thus demonstrating concurrent validity (Nolan et al, 2008). 
Table 2: Frequency of NMS total scores $(n=72)$

\begin{tabular}{|l|l|}
\hline NMS (Total) & $\mathbf{n}(\%)$ \\
\hline$\leq 6$ (Dependent in mobility) & $16(22.2 \%)$ \\
\hline$>6$ (Independent in mobility) & $56(77.8 \%)$ \\
\hline Mean ( \pm SD) & $7.83( \pm 1.83)$ \\
\hline Median (IQR) & $9(1)$ \\
\hline
\end{tabular}

Table 3: Pre-fracture functional level and post-operative functional outcome correlation.

\begin{tabular}{|l|l|l|}
\hline \multirow{2}{*}{ Assessment Period } & \multicolumn{2}{|l|}{ Spearman Correlations } \\
\cline { 2 - 3 } & Elderly Mobility Scale & $\begin{array}{l}\text { Lower Extremity Functional } \\
\text { Scale }\end{array}$ \\
\cline { 2 - 3 } & NMS & NMS \\
\hline Discharge & $0.66^{* *}$ & $0.69 * *$ \\
\hline $\begin{array}{l}\text { Six weeks post } \\
\text { discharge }\end{array}$ & $0.66^{* *}$ & $0.65^{* *}$ \\
\hline
\end{tabular}

**Correlation is significant at the $(p \leq 0.01)$

The Lower Extremity Functional Scale (LEFS) has been used to evaluate the functional activity level of a patient with a disorder of one or both lower extremities (Binkley et al, 1999). The LEFS is a questionnaire containing 20 questions about a person's ability to perform everyday tasks. The questionnaire starts off by asking the patient: "Today would you have any difficulty with:" followed by a list of functional activities. These activities are scored from zero (extreme difficulty) to four (no difficulty). The scale has columns which are summed to get a score. The maximum score is 80 . The LEFS has shown to be both reliable and valid. Construct validity was determined by comparing the LEFS to the physical function subscale $[\mathrm{r}=.80(95 \%$ lower limit CI $=.73)]$ and the physical component score $[\mathrm{r}=.64(95 \%$ lower limit $\mathrm{CI}=.54)]$ of the SF-36. Intra-rater reliability was $\mathrm{r}=.86(95 \%$ lower limit $\mathrm{CI}=.80)$ (Binkley et al, 1999) The lower the score the greater the disability. For the purpose of this study, the LEFS was modified for cultural appropriateness. The activity 'walking a mile' may not be relatable to all elderly South African individuals and was therefore modified accordingly.

\section{PROCEDURE}

\section{Pre-operative assessment:}

Patients were interviewed by the researcher using the New Mobility Score (NMS). The results obtained from the participant for the NMS were verified by a caregiver of the participant.

\section{Discharge and six weeks post dis-} charge assessment:

The EMS assessments were carried out by the researcher in the physiotherapy gym. Lying to sitting and sitting to lying were the first two activities that participants performed. These two tests were performed on a standard plinth in the physiotherapy gym. Thereafter, the participant was assessed on their ability to rise to standing from a $47 \mathrm{~cm}$ (19in) chair in less than three seconds allowing the use of upper limbs. The ability to maintain an upright standing position with or without the use of upper limbs to steady self was also assessed. Participants were then instructed to mobilise, the scoring for gait was based on the type of assistance required to walk, not the distance walked. The participant was then timed walking over a distance of 6 meters, at their normal speed, using their usual walking aid. A maximum score was given for a time of under 15 seconds. Finally the participant was required to reach forward beyond an arm's length while maintaining a fixed base of support. A maximum score was attained for a functional reach of $20 \mathrm{~cm}$.

Participants were also interviewed using the LEFS. This tool was used to capture data on lower limb function.

\section{Data analysis}

Data were analysed using IBM SPSS Version 19. Descriptive statistics were used to reduce the participants' demographic data. Data have been presented using frequency tables, means and standard deviation or medians and interquartile ranges depending on the distribution of the data. The Pearson correlation coefficient (r) was used to test if a linear relationship existed between two variables. A multiple regression analysis was used to determine associations. The study was set at $\mathrm{p} \leq 0.05$ level of significance and $95 \%$ confidence interval $(\mathrm{CI})$.

\section{RESULTS}

Of the 90 participants recruited for the study at baseline, $\mathrm{n}=50$ (69.4\%) participants were female and $n=22(30.6 \%)$ were male. The mean age of the participants was 76 years $(\mathrm{SD} \pm 9.54)$. The minimum age was 60 years and the maximum was 95 years. From the 90 entrants at baseline, only 72 participants were eligible for analysis and were thus analysed. The $n=18$ were excluded from analysis for various reasons as presented in figure 1.

Table 2 presents the frequency of NMS scores and the percentages of each total score $(n=72)$.

Of the 72 participants who completed the study, 16 participants were dependent in mobility $(22.2 \%)$ compared to the 56 participants $(77.8 \%)$ that were independently mobile. More specifically $63 \%$ of the participants scored a total of nine on the NMS, indicating that more than half of the participants were independent with mobility without an assistive device prior to sustaining a hip fracture. And almost $80 \%$ of the participants were independent with mobility with the occasional use of an assistive device. The mean score for the NMS was $7.83(\mathrm{SD} \pm 1.83)$. The median was $9(\mathrm{IQR}=1)$. 
The correlation between pre-fracture functional level and early post-operative functional outcome is shown in Table 3.

Pre-fracture functional level is strongly related to the post-operative functional level at discharge and six weeks post discharge using the EMS $(\mathrm{r}=0.66, \mathrm{r}=0.67)$ and LEFS $(\mathrm{r}=0.69$, $\mathrm{r}=0.65)$.

The NMS $(\beta=1.39, \mathrm{p}=0.0001)$ assessed pre-operatively is a strong predictor of early post-operative functional outcome using the EMS at discharge. The NMS $(\beta=2.35, \mathrm{p}=$ 0.002 ) is also a strong predictor of early post-operative functional outcome using the LEFS at discharge. Figures 2 and 3 depict the results of the multiple regression analysis using the EMS and LEFS at discharge. These figures clearly indicate that the distribution of residuals on the histogram falls suitably, thus indicating that the residuals are normally distributed. Scatterplots and P-P plots depicting these results also indicate normal distribution of residuals. Only the histograms are presented in this article. figures 2 and 3

Similarly the NMS $(\beta=1.3, \mathrm{p}=$ 0.001 ) assessed pre-operatively is a strong predictor of early post-operative functional outcome using the EMS at six weeks post discharge. The NMS $(\beta=2.08, p=0.03)$ is also a strong predictor of early post-operative functional outcome using the LEFS at six weeks post discharge. Figures 4 and 5 are histograms depicting the results of the multiple regression analysis using the EMS and LEFS at six weeks post discharge. These figures clearly indicate that the distribution of residuals falls suitably, thus indicating that the residuals are normally distributed. 4 and 5 here]

In summary, the results reveal that majority of participants were independent in mobility prior to sustaining a fracture. The pre-fracture functional mobility is a strong predictor of early post-operative functional outcome.

\section{DISCUSSION}

In this study, pre-fracture functional mobility was the strongest determinant of early post-operative functional outcome. These results are similar to those of Kristensen et al, (2010), who found that

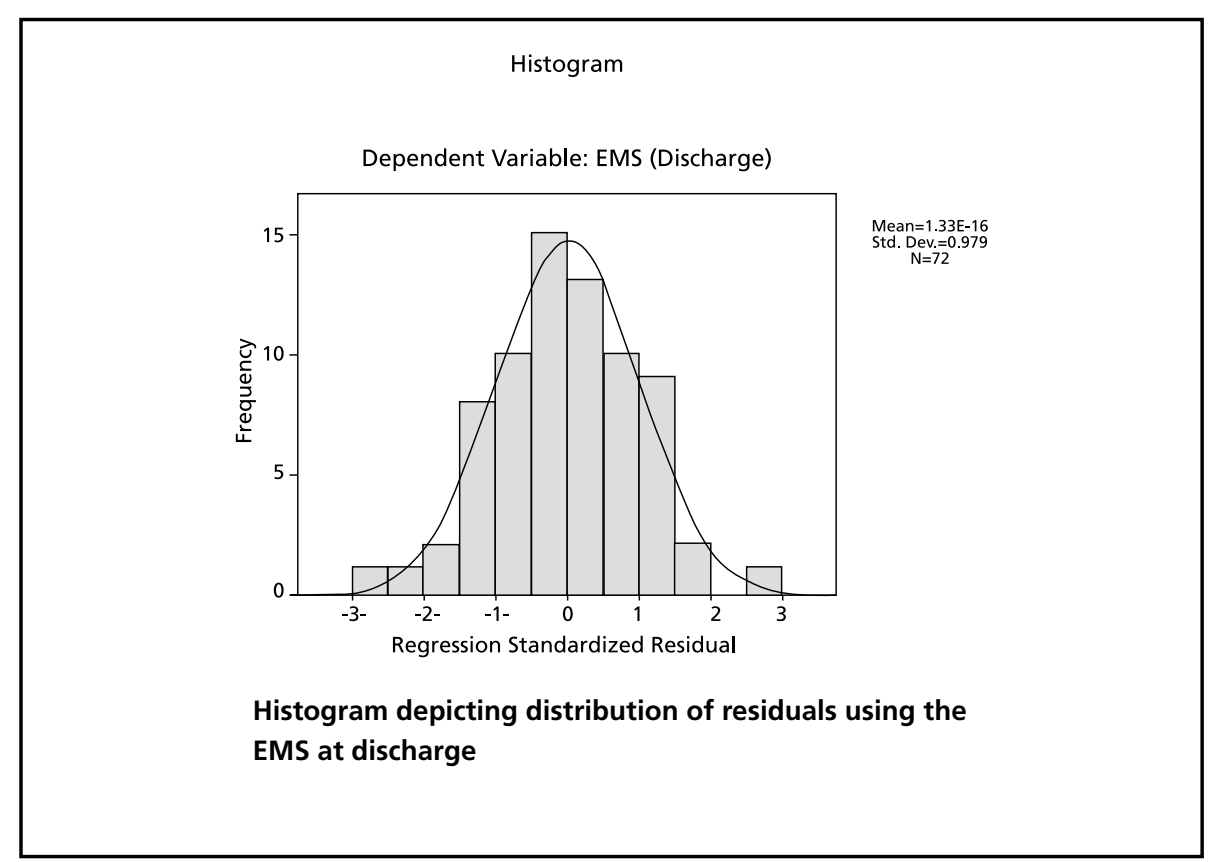

Figure 2: Histogram depicting the distribution of variables using the Elderly Mobility Scale at discharge.

Histogram

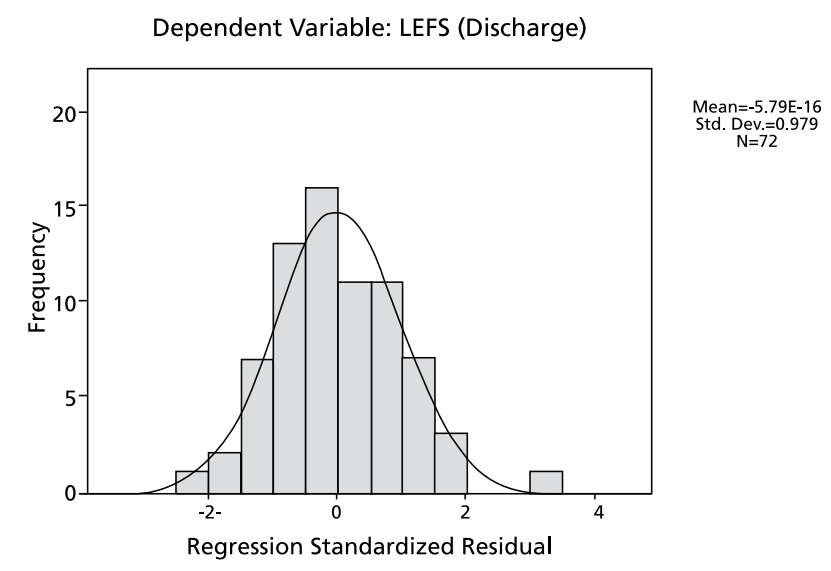

Histogram depicting distribution of residuals using the LEFS at discharge

Figure 3: Histogram depicting the distribution of variables using the Lower Extremity Functional Scale at discharge.

the pre-fracture functional level is a strong predictor of in-hospital outcome in elderly patients with a hip fracture. In the study by Kristensen et al, (2010) the NMS was used to determine prefracture functional level and participants were admitted from their own homes. Similar findings have also been reported by Penrod et al, (2008) and Parker and Palmer, (1993).

Kristensen et al, (2010) reported that $50 \%$ of participants used a walking aid prior to the fracture. In this study only $20 \%$ of participants occasionally required an assistive device. Of the 72 participants who completed this study, $22 \%$ of participants were dependent in mobility while $78 \%$ of participants were independently mobile. In Kristensen et al, (2010) study, 47\% of participants were dependent with mobility while $53 \%$ were independent. These differences could be due to the fact that the mean age in the study by Kristensen et al, (2010) 


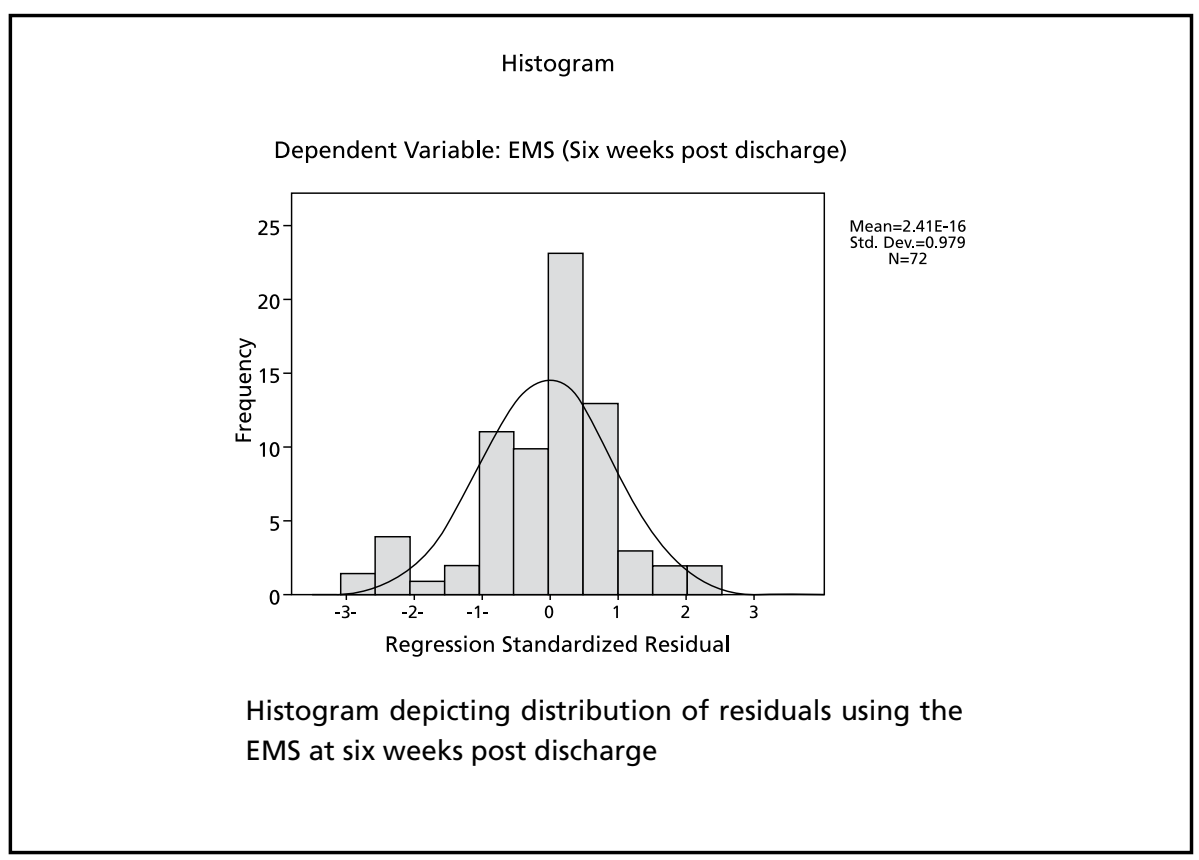

Figure 4: Histogram depicting the distribution of variables using the Elderly Mobility Scale at six weeks post discharge

\section{Histogram}

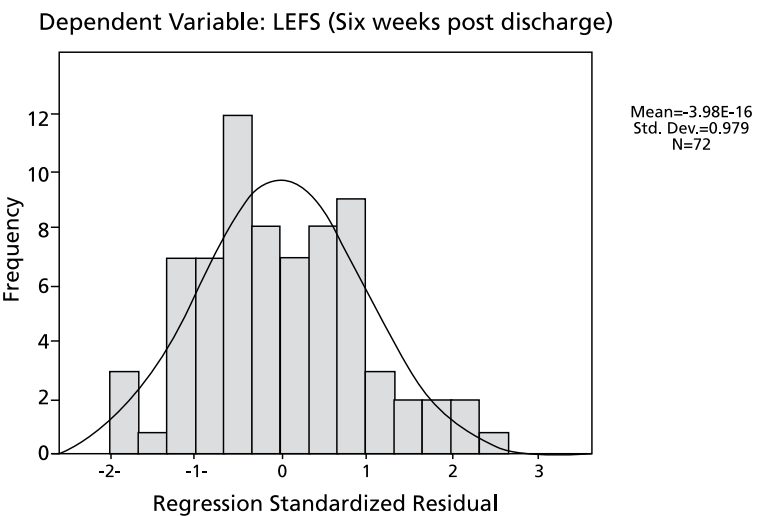

Histogram depicting distribution of residuals using the LEFS at six weeks post discharge

Figure 5: Histogram depicting the distribution of variables using the Lower Extremity Functional Scale at six weeks post discharge

was 81 years, whereas in this study the mean age was 76 years.

Pre-fracture functional mobility was found to be the strongest determinant of post-operative functional outcome. By ensuring that mobility prior to sustaining a hip fracture is optimal, elderly patients are more likely to have better outcomes.

\section{CONCLUSION}

Hip fracture is a common, serious injury that occurs predominantly in the elderly especially necessary for the patient that presents with poor pre-fracture function.

\section{IMPLICATIONS FOR PRACTICE}

The finding that, poor pre-fracture functional level affects the early post-operative functional outcome following a hip fracture in the elderly has important implications for clinical practice.

More intensive rehabilitation should be implemented in order to facilitate a more rapid return to function in this high risk elderly population. Those patients who adopt a sedentary lifestyle or remain bed-ridden following surgical fixation of a hip fracture impact on the financial burden of health care. Therefore, those patients presenting with poorer pre-fracture function should be especially encouraged to participate in intensive rehabilitation.

\section{REFERENCES}

Binkley JM, Stratford PW, Lott SA, Riddle DL, 1999 The Lower Extremity Functional Scale (LEFS):Scale development, measurement properties, and clinical application. Physical Therapy 79(4): $371-382$

Foss NB, Kristensen MT, Kehlet H, 2006 Prediction of postoperative morbidity, mortality and rehabilitation in hip fracture patients: the cumulated ambulation score. Clinical Rehabilitation 20: 701-708

Hagino T, Ochiai S, Sato E, Watanabe Y, Senga S, Haro H, 2011 Prognostic prediction in patients with hip fracture: risk factors predicting difficulties with discharge to own home. Journal of Orthopaedic Traumatology 12: 77-80

Hershkovitz A, Kalandariov Z, Hermush V, Weiss R, Brill S, 2007 Factors affecting shortterm rehabilitation outcomes of disabled elderly patients with proximal hip fracture. Archives of physical medicine and rehabilitation88: 916-921

Holt EM, Evans RA, Hindley CJ, Matcalfe JW, 19941000 femoral neck fractures: the effect of pre-injury mobility and surgical experience on outcome. Injury 25: 91-95

Holt G, Smith R, Duncan K, Hutchison JD, Gregori A, 2008 Outcome after surgery for the treatment of hip fracture in the extremely elderly. Journal of Bone and Joint Surgery America 90: 1899-1905

Kalula S, 2012 Prevalence and problem of falls in older persons.Institute of Ageing in Africa, University of Cape Town (unpublished) 
Kristensen MT, Foss NB, Ekdahl C, Kehlet H, 2010 Prefracture functional level evaluated by the New Mobility Score predicts in-hospital outcome after hip fracture surgery. Acta Orthopaedica 81(3): 296-302

Kristensen MT, Foss NB, Ekdahl C, Kehlet H, 2010 Prefracture functional level evaluated by the New Mobility Score predicts in-hospital outcome after hip fracture surgery. Acta Orthopaedica 81(3): 296-302

Nolan JS, Remilton LE, Green MM, 2008 The reliability and validity of the Elderly Mobility Scale in the acute hospital setting. The Internet Journal of Allied Health Sciences and Practice http://ijahsp.nova.edu Volume 6 No. 4 ISSN 1540580XAccessed 01/03/2011

Parker MJ, Palmer CR, 1993 A new mobility score for predicting mortality after hip fracture. Journal of Joint and Bone Surgery 75-B: 797-798

Penrod JD, Litke A, Hawkes WG, Magaziner J, Doucette JT, Koval KJ, Silberzweig SB, Egol KA, Sui AL, 2008 The association of race, gender and co-morbidity with mortality and function after hip fracture. The Journals of Gerontology 63A: 867-872

Smith R, 1994 Validation and Reliability of the Elderly Mobility Scale. Physiotherapy 80, 11: 744-747

World Health Organization, 2007 WHO global report on falls prevention in older age. Geneva: World HealthOrganizationhttp://www.who.int/ageing/ publications/Falls_prevention7March.pdfAccessed $31 / 10 / 2012$

Williams A, Jester R, 2004 Delayed surgical fixation of fractured hips in older people: impact on mortality. Journal of Advancing Nursing 52, 1: 63-69 